\title{
Clinical Characteristics of Brain Abscess in a Developing Country, a Number of Cases
}

\author{
Juárez-Rebollar Daniel $^{1 *}$, Juárez-Rebollar Alejandra Giselle ${ }^{2}$, Sosa-Martínez María Del Rosario ${ }^{3}$, Valdés-García Javier ${ }^{3}$, \\ Broc-Haro Guy Gilbert ${ }^{4}$ \\ ${ }^{1}$ PGY4 Neurosurgery, Regional Hospital Lic. Adolfo López Mateos, México \\ ${ }^{2}$ OMFS National Medical Center "Siglo XXI", Mexico City \\ ${ }^{3}$ MD Neurosurgery, Hospital Lic. Adolfo López Mateos. Mexico City \\ ${ }^{4}$ Director of the Department of Neurosurgery, Hospital Lic Adolfo López Mateos, Mexico City
}

Article History

Received: 11.03.2021

Accepted: 15.04.2021

Published: 22.04.2021

Journal homepage:

https://www.easpublisher.com

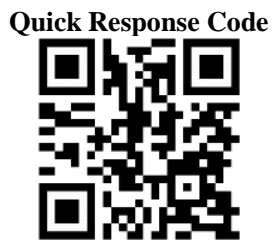

Abstract: Brain abscess has been considered a rare infection; it is characterized by a collection of pus in the brain parenchyma. Different studies talk about the management and medical treatment with antibiotic regimens (at the beginning of therapy, the antibiotic is chosen empirically), among the most used are vancomycin, thirdgeneration cephalosporins, clindamycin, trimethoprim-sulfamethoxazole, aztreonam, metronidazole, fluoroquinolone, and fluconazole when it is a fungal-type infection, and linezolid has shown promising results. Surgical management depends on the size, location, and characteristics of the brain abscess; it is suggested in the literature for small brain abscess do the aspiration and eradicates the purulent material, and in a big abscess $(>2.5 \mathrm{~cm})$ performs craniotomy and resection. A decrease in the degree of mortality related to brain abscess has been reported. That is why this study is presented, carried out over three years (2018 -2021) showing the results obtained, as well as the frequency of patients with brain abscess treated in the service of neurosurgery, of the Regional Hospital "Lic. Adolfo López Mateos", in Mexico City. A series of cases is presented showing the evaluation and diagnosis protocol used in Neurosurgery and the clinical-epidemiological characteristics that the patients gave.

Keywords: Brain abscess, surgical management, cerebritis, infection.

Copyright (C) 2021 The Author(s): This is an open-access article distributed under the terms of the Creative Commons Attribution 4.0 International License (CC BY-NC 4.0) which permits unrestricted use, distribution, and reproduction in any medium for non-commercial use provided the original author and source are credited.

\section{INTRODUCTION}

Brain abscess are defined as a collection of pus located in the brain parenchyma, the presence of a triad consisting of fever, headache, and focal signs. Brain abscess are a rare pathology with a long evolution that has had better treatment thanks to medical advances, antibiotic therapy, scientific and technological advances. Only on some occasions, it causes death [1]. Since the 19th century, series have been reported to provide more information on brain abscess [1]. Few significant case series are known, and most are from studies carried out over long periods of time [2-6]; for example: in 1973, Kao et al. reported some of the clinical characteristics of 26 cases of brain abscess with surgical intervention. However, not all the results of hospital management were obtained, mainly $[2,3]$. In 1980, small series of subjects related to this infection were also reported [1]. There is a series of 16-year-old cases (1989-2005) carried out in Chile [4] where variables such as age, gender, risk factors, etiology, clinical manifestations, and location were studied.
These authors were referring that they presented some inconveniences in the data collection. On the other hand, in 2005, in Italy 100 cases of brain abscess were reported in 17 years [5]. Another representative series was carried out in Bangladesh, from July 1999 to June 2013, 162 patients diagnosed with brain abscess were reported in 14 years [6]. However, the majority of investigations in this regard speak about the generalities of the infection [7-11]. Brain abscess can have an idiopathic origin, arise spontaneously, or be associated with factors such as intravenous drug use, congenital heart defects, and infective endocarditis. More cases have been reported in patients with immunosuppression, or with a contiguous focus of spread of the infection to the adjacent central nervous system (infection of otic, dental origin, concerning the paranasal sinuses, among others) [10-14]. The brain abscess is more frequent in men (the literature reports $70 \%$ of the cases in men) and the majority has been young patients $[10,11,15]$. Brain abscess are usually staged in 4 stages $[3,16]$ : (table 1$)$. 
Table-1: Stages of a brain abscess and characteristics

\begin{tabular}{|l|l|l|}
\hline STAGE & EVOLUTION & CHARACTERISTICS \\
\hline Early cerebritis & $1-4$ days & $\begin{array}{l}\text { Neutrophil accumulation, tissue necrosis, edema, Activation } \\
\text { of microglia and astrocytes }\end{array}$ \\
\hline Late cerebritis & $4-10$ days & Lymphocyte and macrophage predominance \\
\hline Early capsular formation & $11-14$ days & $\begin{array}{l}\text { A highly vascular capsule is formed; in this way, the adjacent } \\
\text { tissue is kept safe }\end{array}$ \\
\hline Late capsular formation & $>14$ days & $\begin{array}{l}\text { Maturation of the abscess the capsule thickens (if it is more } \\
\text { than three weeks old), excision can be performed. }\end{array}$ \\
\hline
\end{tabular}

The pathogens related to bacterial brain abscess are associated with different factors, such as the patient's age, medical conditions, previous surgeries, time of evolution, origin, type of infection, etc. [3] Microorganisms most frequently isolated are Viridans Streptococci, Staphylococcus Aureus, Gram-negative bacilli, and anaerobes, however other resident and opportunistic species will be found depending on the origin of the infection [1, 17]. Generally, the imaging studies are correlated to the histopathological stage and the evolution of the infection. In the initial phase (cerebritis), a diffuse hypodense area is observed in the CT scan, which, when viewed with contrast, reveals a halo. Simultaneously, in encapsulation, the lesion will have a more hypodense center (central necrosis with liquefaction, surrounded by a hyperdense halo that is the capsule) and intensifies contrast material is applied 18. In the MRI, cerebritis looks like a hypointense image on $\mathrm{T} 1$ and hyperintense on $\mathrm{T} 2$ with ring enhancement. When they progress and encapsulate, they show a hypointense center on $\mathrm{T} 1$ and hyperintense on T2 with an isointense to moderately hyperintense on $\mathrm{T} 1$ and hyperintense on the $\mathrm{T} 2$ capsule. By adding gadolinium contrast, the capsule has more intensity in the halo and its defined edges [19]. The treatment is usually dual, surgical, and medical (only some cases can be treated with medical therapy only) [19, 20]. Antibiotic treatment is started empirically with a high dose of a combination of antibiotics; among the most used are: cephalosporins (ceftriaxone/cefuroxime), meropenem, flucloxacillin, vancomycin, and metronidazole. The scheme is maintained for 4-6 days and is subsequently modified to antibiotic sensitivity and is maintained for 4-8 weeks according to the patient's response. A low-dose corticosteroid is used to control perilesional edema (which occurs in the first 5-7 days). In some patients, anticonvulsants are also added [16]. Surgical management is based on aspiration or resection of lesions with a diameter $>2.5 \mathrm{~cm}$, depending on the size of the lesion [1]. This can be performed through a burr hole, craniotomy, or by stereotaxic surgery (invasive procedure with less postoperative morbidity and is used for diagnosis and treatment); with the techniques mentioned above, it is possible to perform drainage or resection of the abscess, and the installation of an external ventricular drain in severe cases with ventricular eruption [4]. With the help of imaging studies, the development of surgical techniques and the use of antimicrobials with better penetration into the CNS has improved the prognosis of patients with brain abscess $[4,21]$.

\section{NUMBER OF CASES}

The following series of cases is a compilation of the observation for three years (2018-2021), reporting the frequency of patients with a diagnosis of brain abscess treated in the neurosurgery service of the Regional Hospital "Lic. Adolfo López Mateos". A total of 13 patients were obtained. The evaluation and diagnosis protocol that was used was: the clinical correlation, the antecedents, and the subsequent imaging evaluation, a simple and contrasted skull tomography was performed, as well as magnetic resonance imaging with spectroscopy and diffusion sequence; given the high suspicion of the lesion, decided to carry out a surgical procedure. For this, took the location of the lesion, accessibility, and volume took the place of the lesion, accessibility, and volume into account, as well as the maximum diameter, taking as a cut-off point $(2.5 \mathrm{~cm})$ were treated with a triple antibiotic scheme and later with monoschema of intravenous linezolid for two weeks and later for six weeks orally with monitoring in the outpatient clinic, as illustrated in table 2; As observed in our hospital center, men are predominant in a 1.6: 1 ratio, with a median age of 51 years [19-77 years], the most frequent comorbidity was Systemic Arterial Hypertension; The initial symptom was a headache, reported in $53 \%$ of the cases, only in two instances the primary infectious focus was not identified, as regards cryptogenic, the rest of the cases located the origin, the pulmonary being more frequent by $46 \%$. The hemisphere with the most significant involvement was the right, the regions with the most involvement were the frontal lobe $69 \%$, and the average volume of the lesion was $27.6 \mathrm{cc}$, with a maximum diameter of 4.65 , with expansive lesions (Image 1). 

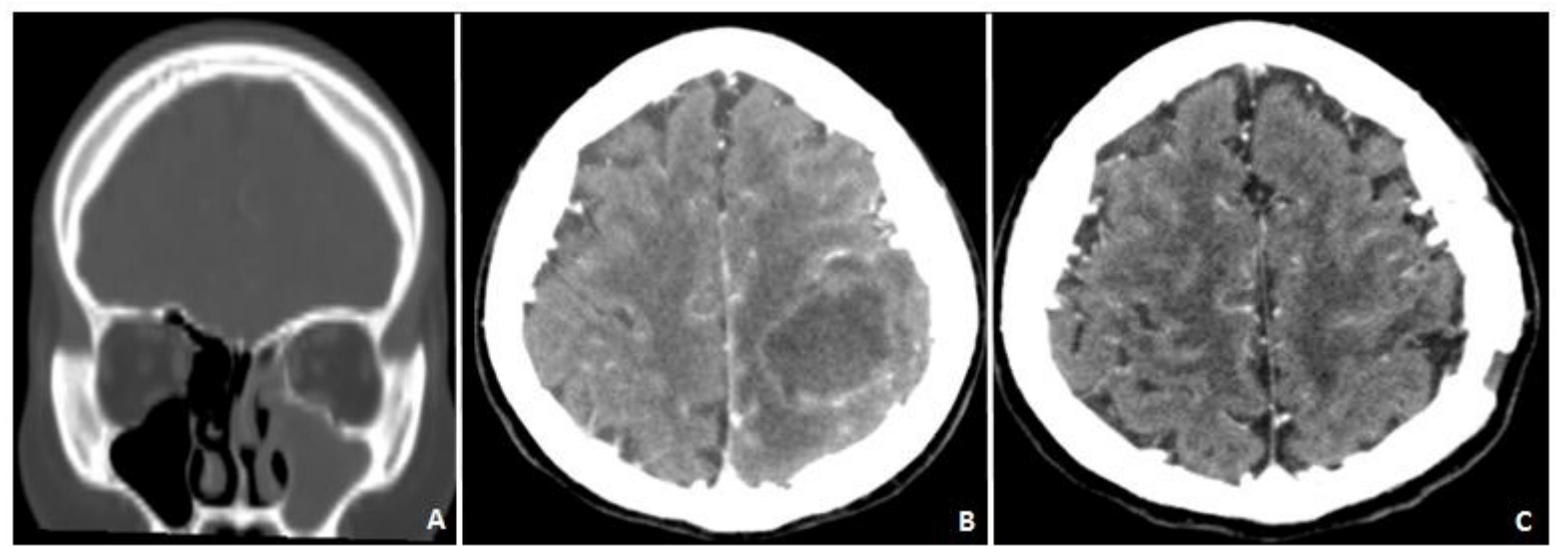

Image-1: Representative case. Twenty-nine years female, with the onset of diabetic ketoacidosis, after resolving the crisis, she presents headache, right hemiparesis, and seizure; in the emergency room, take a CT. Panel A. Bone window coronal plane observed a hyperdensity inside of the sinuses. Panel B contrast axial plane, heterogeneous image with enhanced of the lession parietal and frontal extension. Panel $\mathrm{C}$ axial plane contrast $\mathrm{CT}$ changes posttreatment by craniotomy and resolution of the abscess

Only in two cases was puncture of the lesion and subsequent resection performed, the most frequent procedure was resection of the capsule, all patients received empirical antibiotic management with a triple scheme with metronidazole, vancomycin, and ceftriaxone, once with cultures and identified microorganism, changed the antibiotic plan to monoschema with linezolid, intravenous impregnation and later orally, all patients underwent a controlled study at three months, six months and after one year (Image 2), only three were reported cases of mortality from systemic complications; 10 cases with notable improvement in the ranking scale.
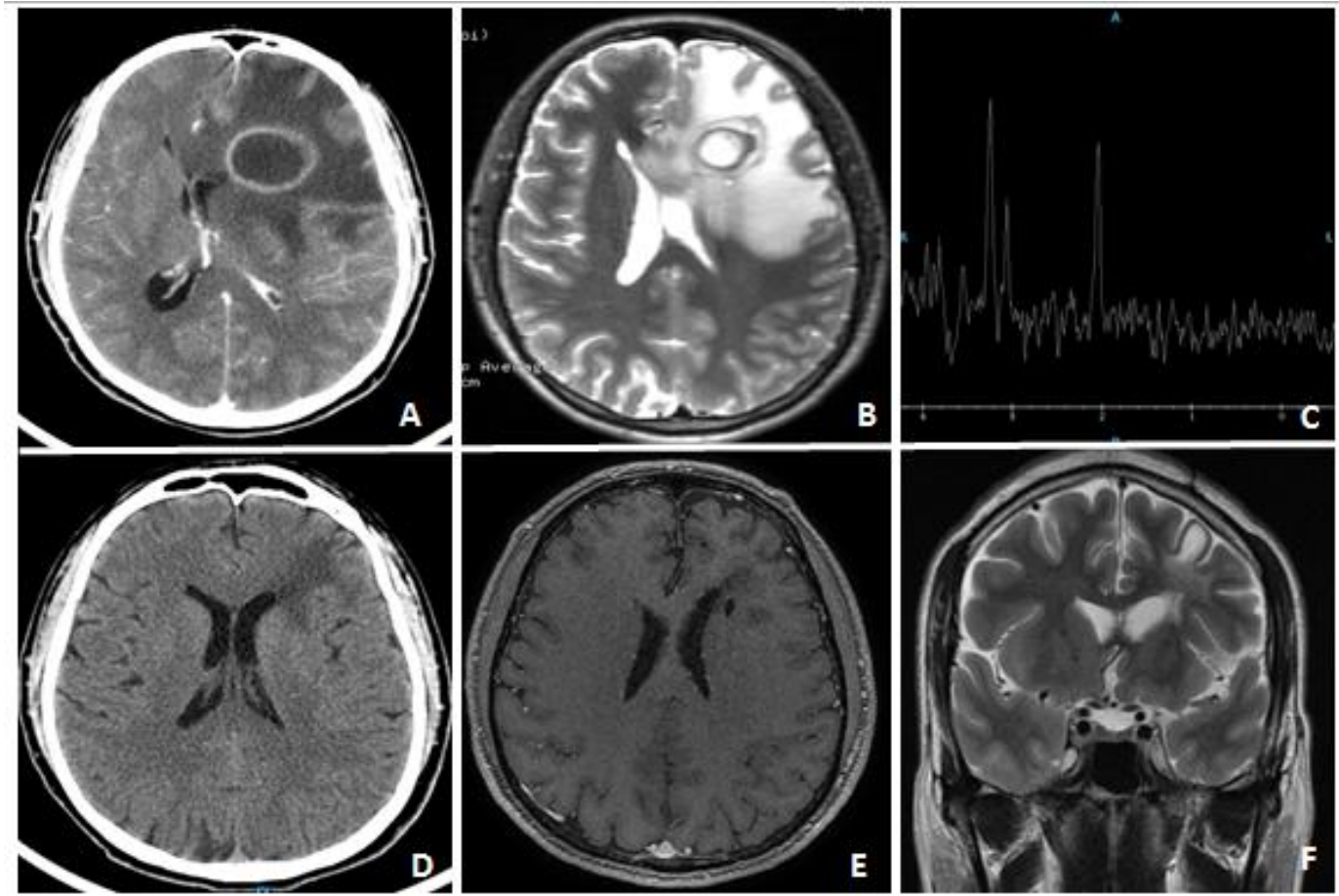

Image-2: Representative case. Panel A. CT axial plane shows a lesion with enhanced ring sign and hypodense area inside. Panel B. MRI T2 axial plane with important edema perilesional in the frontal lobe with mass compression to the left horn of ventricle. Panel C. Spectroscopy with increased amino-acid peak, the elevation of the lipid/lactate peak. Panel D. Postsurgery CT with complete resolution at three months. Panel E and F MRI axial and coronal plane T1 and T2 sequences, at six months and one year of following 


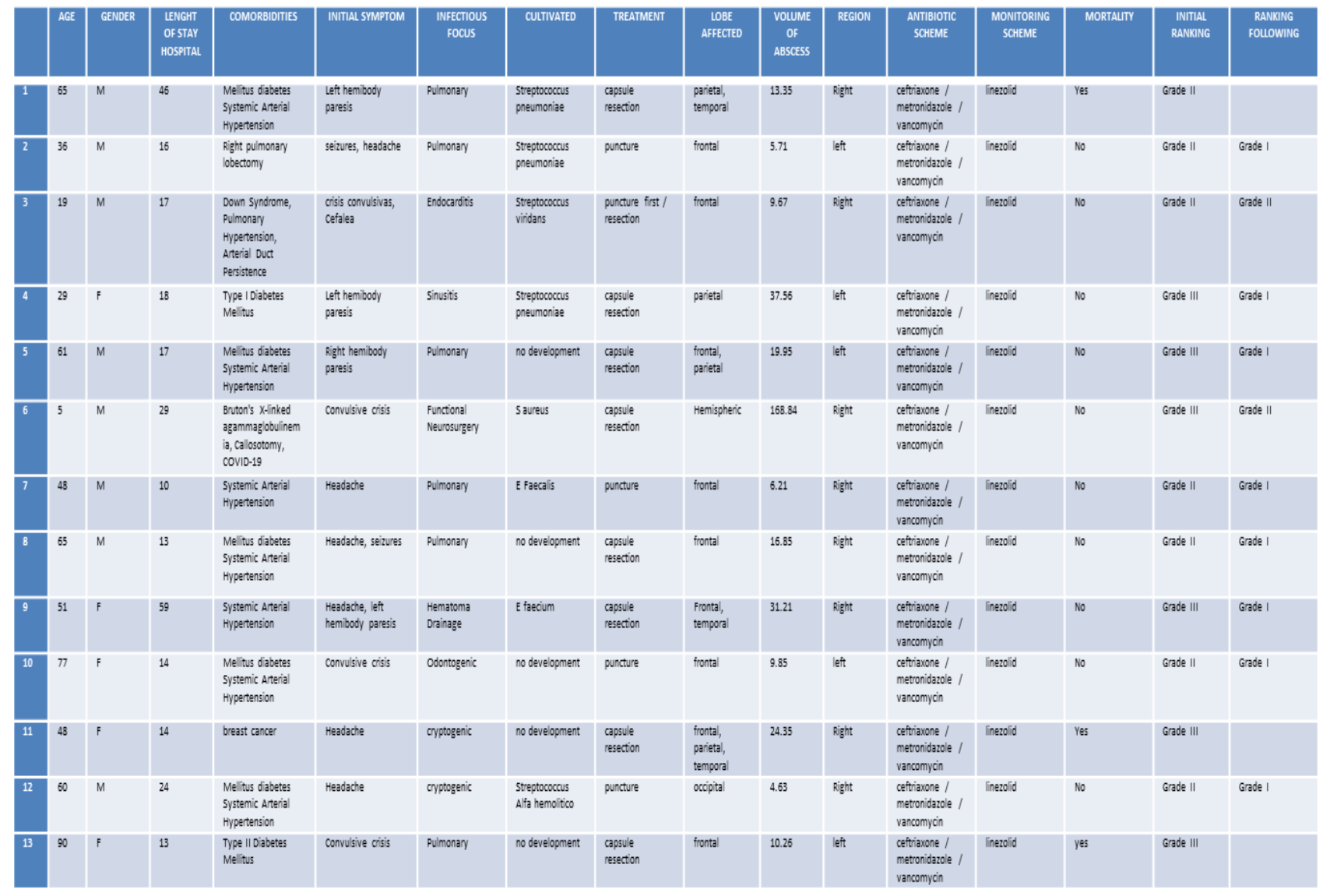

\section{DISCUSSION}

The cases presented in this study were 13 patients with a diagnosis of brain abscess in 3 years, which represents arithmetic mean of 4.33 patients per year; compared with other cases series studies such as that of Fica et al. four who reported 30 cases in 16 years or large series such as Lu, Chang et al. three who describe a series of 123 patients in 15 years, or the study by Tonon et al. five who reported 100 patients with the same diagnosis in 17 years, or by Hossain et al. [16] who reported 162 patients in 14 years; whose means of reported cases of brain abscess range between 1.87 (minimum) and 11.57 (maximum). In different studies, they mention that clinical correlation with the image is required (with computed tomography and magnetic resonance imaging) [4, 7-9, 11]. This study presented was not the exception, since a diagnostic protocol with clinical data, simple and contrasted phase tomography, was performed on all patients; Magnetic resonance with spectroscopy and diffusion sequence. Among the patients' clinical characteristics in this study, they were predominantly male, which coincides with that reported by Lu, Chang et al. [3], Hossain et al.[16], Menon [23], Kaczorowskay [24] Sharon, et al. [25] On the other hand, one of the differences found in this study for others was age since the current series of cases had a median of 51 years. In other articles, it is mentioned that brain abscess has generally been reported among the 2 nd and 3rd decade of life [8, 23,
24] although there are studies that report patients with brain abscess in the 6th decade $[10,11]$ like this study. The most frequent comorbidity was systemic arterial hypertension, unlike other articles such as that of Alvis et al. [8], Menon et al. [23], those who express greater frequency in diabetic patients; However, in most studies, the association of brain abscess with a state of immunosuppression of the patient is reported as in this one $[8,10,11,23,25]$. In this study, the origin of brain abscess was $46 \%$ pulmonary and a little more than $50 \%$ due to other causes (including endocarditis, odontogenic, sinusitis, cryptogenic, among others); Concerning other studies, it is mentioned that the most common route of dissemination is hematogenous, related to pneumonia, empyemas, and endocarditis. Besides, it is mentioned that other routes are by direct dissemination (such as head trauma, facial procedures, placement of catheters, or ventricular drains, among others.) [26, 27, 28]. One of the considerations regarding the site most affected by a brain abscess is that other authors highlight the location in decreasing order: Fronto-temporal, Fronto-parietal, Cerebellar, and Occipital lobe [29]. Morgan et al. [30] They reported in a series of 86 cases 22 brain abscess in the parietal lobe, 21 in the frontal, 18 in the temporal, five occipital, four hemispherical, 12 cerebellar, and six multiple [30]. In this article, the most affected hemisphere was the right and $69 \%$ of the cases in the frontal lobe, which coincides with that mentioned by Shachor et al. [31]. Regarding the average volume obtained of purulent 
material, it was $27.6 \mathrm{cc}$, as for the measurements of the lesions, they were wide of approximately $4-5 \mathrm{~cm}$ in diameter, and the most used treatment was by resection of the capsule. In this study, mortality of less than $50 \%$ of the cases is reported, with only three deaths. In other studies carried out in developing countries (the incidence of brain abscess is 4-11 cases per million inhabitants), mentioning that brain abscess corresponds to less than $10 \%$ of all intracranial masses (reporting an average of $8 \%$ in developing countries). In developed countries, they are present at $1-2 \%[8,32]$. Also, the incidence of brain abscess is higher in underdeveloped countries compared to the cases reported in developed countries. For example, Bhattia et al. reported that $8 \%$ of intracranial lesions seen in a hospital in India were focal pyogenic collections. While the incidence in countries such as the United States reported between 1500 and 2500 cases per year of brain abscess, mentioning a higher prevalence in men 3:1.33 In Latin America, the annual incidence is 1600 to 2500 cases a year with greater affection in the second decade of life [34]. Regarding the number of cases reported according to hospital admissions, in industrialized countries, it is 1: 10,000. In Latin America, it is 1.5 admissions (hospital) per year per 1606 hospitalized [4]. Brain abscess have increased in frequency; some authors mention that it is possibly related to the increase in the number of immunocompromised patients with such abscess caused mainly by opportunistic infections mortality ranges between 5 and $15 \%$ of cases, except in intraventricular rupture of brain abscess [33, 35], in which mortality ranges between $38 \%-84 \%$, with high rates of disability in survivors [36]. Even though the incidence of Brain abscess has decreased considerably, it continues to be a very significant public health problem, not only for the patient but also for hospital staff, the patient's family, the community and the State, mainly due to secondary complications, which lead to a prolonged hospital stay, use of more expensive antibiotics and re-surgical interventions.

\section{Conclusions}

Brain abscess is still considered an infection that has to be treated urgently. The importance of reporting series of cases allows showing the number of cases that occur at a particular time. The characteristics they have since most of the reports are have been carried out in developed countries. They could influence the frequency of cases, as well as make a comparison with what was found by other authors in other studies. With this, a broader perspective is obtained mainly in terms of progress, treatment, and epidemiological data, which at the same time it, allows knowing the population under study in developing countries. This study shows some differences to other studies, mainly in that mortality was not as high as in studies carried out a few decades ago, where brain abscess was considered to have a high mortality rate, management, and treatment, as well as An early diagnosis, has allowed this rate of deaths related to the disease to be reduced over the years.

\section{Funding}

We have not received financing nor do we have sponsors to carry out this study.

\section{Competing interest statement}

The authors have declared no competing interest. Any institution has not funded the study. We confirm all relevant ethical guidelines have been followed.

\section{REFERENCES}

1. Calfee, D., Wispelwey, B. (2000). Brain abscess. Semin Neurol, 20, 353-60.

2. 高明見. (1973). Brain-abscess: a clinical analysis of 26 cases with surgical treatment (Doctoral dissertation, 東京医科歯科大学).

3. LU, C. H., Chang, W. N., LIN, Y. C., TSAI, N. W., Liliang, P. C., SU, T. M.,... \& WU, J. J. (2002). Bacterial brain abscess: microbiological features, epidemiological trends and therapeutic outcomes. Qjm, 95(8), 501-509.

4. Fica, C. A., Bustos, G. P., \& Miranda, C. G. (2006). Brain abscess: about a clinical series of 30 patients. Revista chilena de infectologia: organo oficial de la Sociedad Chilena de Infectologia, 23(2), 140-149.

5. Tonon, E., Scotton, P. G., Gallucci, M., \& Vaglia, A. (2006). Brain abscess: clinical aspects of 100 patients. International journal of infectious diseases, 10(2), 103-109.

6. Chowdhury, F. H., Haque, M. R., Sarkar, M. H., Chowdhury, S. M., Hossain, Z., \& Ranjan, S. (2015). Brain abscess: surgical experiences of 162 cases. Neuroimmunology

and Neuroinflammation, 2, 153-161.

7. Daniel, J. R., Giselle, J. R. A., del Rosario, S. M. M., Christopher, M. A., Gilbert, B. H. G., \& Daniel, J. R. (2021). Brain abscess Review. International Journal, 4(1), 707.

8. Alvis-Miranda, H., Gutiérrez-Paternina, J. J., Alcalá-Cerra, G., Castellar-Leones, S. M., \& Moscote-Salazar, L. R. (2013). Brain abscess. Revista Cubana de Neurología y Neurocirugía, 3(2), 162-171.

9. Molina, G., Armijo, Y., Alvarado, M. (2010). Brain Abscess. Mexican Journal of Neuroscience January-February, 11(1): 63-70

10. Brouwer, M. C., Tunkel, A. R., McKhann, G. M., \& van de Beek, D. (2014). Brain abscess. New England Journal of Medicine, 371(5), 447-456.

11. Brouwer, M. C., \& van de Beek, D. (2017). Epidemiology, diagnosis, and treatment of brain abscesses. Current opinion in infectious diseases, 30(1), 129-134.

12. Tandon, S., Beasley, N., \& Swift, A. C. (2009). Changing trends in intracranial abscesses 
secondary to ear and sinus disease. The Journal of Laryngology \& Otology, 123(3), 283-288.

13. Leroy, O., Georges, H., Devos, P., Bitton, S., De Sa, N., Dedrie, C., ... \& Preau, S. (2015). Infective endocarditis requiring ICU admission: epidemiology and prognosis. Annals of intensive care, 5(1), 1-8.

14. Sonneville, R., Ruimy, R., Benzonana, N., Riffaud, L., Carsin, A., Tadie, J. M., ... \& Tattevin, P. (2017). An update on bacterial brain abscess in immunocompetent patients. Clinical Microbiology and Infection, 23(9), 614-620.

15. Widdrington, J. D., Bond, H., Schwab, U., Price, D. A., Schmid, M. L., McCarron, B., ... \& Ong, E. (2018). Pyogenic brain abscess and subdural empyema: presentation, management, and factors predicting outcome. Infection, 46(6), 785-792.

16. Chowdhury, F. H., Haque, M. R., Sarkar, M. H., Chowdhury, S. M., Hossain, Z., \& Ranjan, S. (2015). Brain abscess: surgical experiences of 162 cases. Neuroimmunology and Neuroinflammation, 2, 153-161.

17. Sofianou, D., Tsakris, A., Selviarides, P., Foroglou, G., \& Sofianos, E. (1996). Etiological agents and predisposing factors of intracranial abscesses in a Greek university hospital. Infection, 24(2), 144-146.

18. Enzmann, D. R., Britt, R. H., \& Placone, R. (1983). Staging of human brain abscess by computed tomography. Radiology, 146(3), 703708.

19. Mathisen, G. E., \& Johnson, J. P. (1997). Brain abscess. Clinical Infectious Diseases, 763-779.

20. Mamelak, A. N., Mampalam, T. J., Obana, W. G., \& Rosenblum, M. L. (1995). Improved management of multiple brain abscesses: a combined surgical and medical approach. Neurosurgery, 36(1), 76-86.

21. Roche, M., Humphreys, H., Smyth, E., Phillips, J., Cunney, R., McNamara, E., ... \& McArdle, O. (2003). A twelve-year review of central nervous system bacterial abscesses; presentation and aetiology. Clinical microbiology and infection, 9(8), 803-809.

22. Ntziora, F., \& Falagas, M. E. (2007). Linezolid for the treatment of patients with central nervous system infection. Annals of Pharmacotherapy, 41(2), 296-308.

23. Menon, S., Bharadwaj, R., Chowdhary, A., Kaundinya, D. V., \& Palande, D. A. (2008). Current epidemiology of intracranial abscesses: a prospective 5 year study. Journal of medical microbiology, 57(10), 1259-1268.
24. Kaczorowska, B., Chmielewski, H., Pawełczyk, M., Przybyła, M., Błaszczyk, B., \& Chudzik, W. (2007). The case of multiple brain abscesses conservatively treated. Polski Merkuriusz Lekarski: Organ Polskiego Towarzystwa Lekarskiego, 22(128), 150-153.

25. Sharon, E. (2008). Acute Bacterial Meningitis. Emerg Med Clin N Am; 26(2): 281-317.

26. Caballero, F., Bernabeu, W., Jiménez, M., Pachón, D. (1998). Abscess cerebral. Subdural empyema. Epidural abscess. Suppurative phlebitis of the central veins. Medicine, 7(75): 3476-8.

27. Roos, K. L. (2008). Meningitis, encephalitis, brain abscess, and empyema. Harrison's principles of internal medicine, 2621-2641.

28. Vargas, L., Alvarado, M., Suárez, A. (2018). Cerebral abscess: diagnosis, management, complications and prognosis. Rev. Chil. Neurocirugía, 44: 60-68.

29. Hasbun, R., Abrahams, J., Jekel, J., \& Quagliarello, V. J. (2001). Computed tomography of the head before lumbar puncture in adults with suspected meningitis. New England Journal of Medicine, 345(24), 1727-1733.

30. Morgan, H., Wood, M. W., \& Murphey, F. (1973). Experience with 88 consecutive cases of brain abscess. Journal of neurosurgery, 38(6), 698-704.

31. Shachor- Meyouhas, Y., Bar- Joseph, G., Guilburd, J. N., Lorber, A., Hadash, A., \& Kassis, I. (2010). Brain abscess in children-epidemiology, predisposing factors and management in the modern medicine era. Acta Paediatrica, 99(8), 1163-1167.

32. Sharma, B. S., Gupta, S. K., \& Khosla, V. K. (2000). Current concepts in the management of pyogenic brain abscess. Neurology India, 48(2), 105.

33. Rivero-Garvía, M. (2006). Brain abscess associated with Rendu Osler-Weber disease. Rev Neurol, 43(5): 311-13.

34. Duazuary, Ed. (2016). Differential Diagnosis of Ring Enhancement Brain Lesions in computed tomography and magnetic resonance imaging. searchgate.net/publication/307851529_Diagnostic o_diferencial_de_lesiones_Ring_enhancement_br ains_in_computed_tomography_and_magneti_res onance

35. Varela. (2016). Synchronous presentation of intracranial squamous cell tumor and brain abscess. Report of a case. Rev. Chil. Neurosurgery, 42(3):128-132.

36. González, W. (2000). Central nervous system infections: Brain abscess. Journal of Neuroscience, 3, 480-487.

Cite This Article: Juárez-Rebollar Daniel et al (2021). Clinical Characteristics of Brain Abscess in a Developing Country, a Number of Cases. East African Scholars J Med Surg, 3(4), 84-89. 